\title{
Quality Informatics: The Convergence of Healthcare Data, Analytics, and Clinical Excellence
}

\author{
Nathan A. Coppersmith ${ }^{1}$ Indra Neil Sarkar ${ }^{1} \quad$ Elizabeth S. Chen ${ }^{1}$ \\ ${ }^{1}$ Warren Alpert Medical School and Center for Biomedical \\ Informatics, Brown University, Rhode Island, United States \\ Address for correspondence Elizabeth S. Chen, PhD, Center for \\ Biomedical Informatics, Brown University, Box G-R, Providence, RI \\ 02912, United States (e-mail: liz_chen@brown.edu).
}

Appl Clin Inform 2019;10:272-277.

\section{Background and Significance}

The convergence of electronic health record (EHR) adoption, increased availability of electronic health data, development of analytical techniques, and healthcare payment reform has created an environment ripe for using information technology to improve delivery of healthcare services. ${ }^{1-3}$ The lack of unification has manifested itself in EHR systems used by providers, quality measures developed by different groups, and use of quality measures in different ways by different organizations. ${ }^{4}$ The organization and coordination of formal approaches for using health information systems and their data to improve clinical effectiveness is needed.

Quality informatics is defined here as the discipline that "uses healthcare data collected from healthcare information systems to improve clinical effectiveness." As part of clinical informatics that broadly studies the organization and use of information to improve healthcare delivery, ${ }^{6,7}$ quality informatics is rooted in collecting accurate healthcare quality data, analyzing those data, and applying findings toward quality improvement programs ( - Fig. 1). Coordination of this work allows clinicians, analysts, and researchers to unify, align, and share in the work of connecting large-scale data with improvement in healthcare delivery. These efforts complement and contribute to parallel efforts to using EHR data for clinical decision support, research, and public health. ${ }^{8-10}$

This letter outlines why quality informatics should be recognized, provides an overview of the work of quality informatics, and describes the opportunities and challenges facing the work. Electronic clinical quality measures (eCQMs), part of the Centers for Medicare and Medicaid Services (CMS) Promoting Interoperability EHR Incentive Program, will serve as an exemplar of quality informatics in practice. ${ }^{11}$

\section{The Why}

To allow for coordinated, efficient, and best use of clinical data, quality informatics serves as an umbrella to organize

received

September 13, 2018

accepted after revision

March 6, 2019

formal approaches to utilize healthcare information systems and data to improve clinical effectiveness. Medicare utilizes quality metrics that providers must consistently report as a means to tie quality to data through payments. ${ }^{12}$ However, confusion remains about which quality metrics are essential and how they are used. ${ }^{4}$

Despite the importance of utilizing data from healthcare information systems to improve clinical effectiveness, their use present challenges. Dixon-Woods et $\mathrm{al}^{13}$ describe how data from an electronic prescribing decision support system improves quality and safety indicators. This experience serves as an example, but there is no path for formally expanding this type of work. Quality informatics allows techniques, lessons, and approaches from individual experiences to be incorporated with the experiences of others to create effective approaches for improving healthcare delivery.

eCQMs serve as an example of how unification and coordination is needed to support the calculation of quality metrics. CMS provides a technical framework; however, providers are on their own to write code for calculating an eCQM from their systems. Others describing analytic methodologies involving standards such as the HL7 Quality Data Model highlight the need for sharing of methodologies to calculate eCQMs. ${ }^{11,14}$ Quality informatics could guide how methodologies are catalogued, formalized, and distributed for advancing the ease, reliability, and standardization of eCQM use.

\section{The Work}

The goal of quality informatics is to collect effective healthcare quality data, analyze those data, and apply findings toward quality improvement programs. Quality informatics tasks are grouped into three categories: (1) project management; (2) technical management; and (3) reporting. These areas require different skill sets, but each area is inseparable from the others for quality informatics to be effective and useful. (c) 2019 Georg Thieme Verlag KG
Stuttgart · New York
DOI https://doi.org/

10.1055/s-0039-1685221. ISSN 1869-0327. 


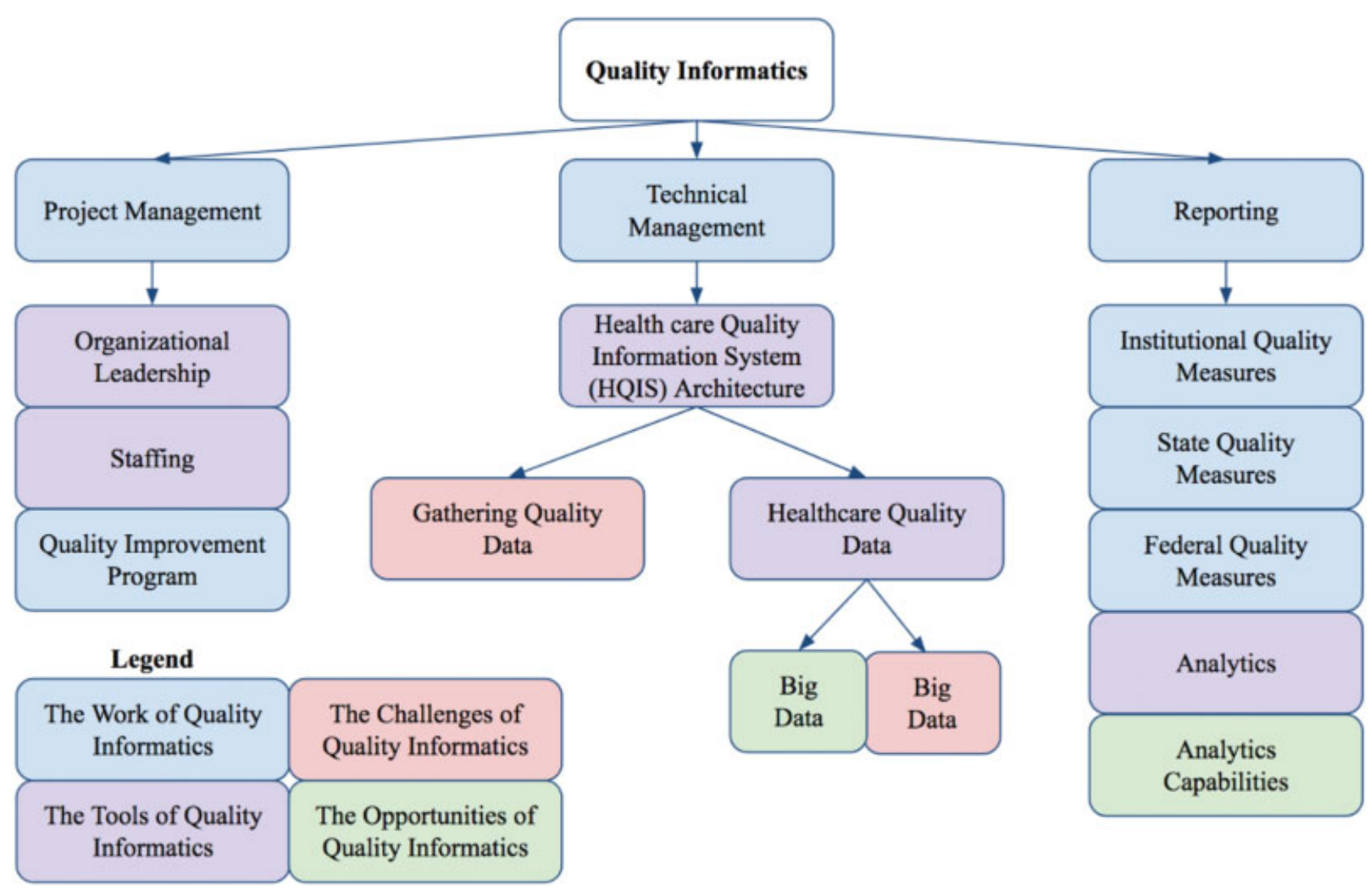

Fig. 1 The work, tools, challenges, and opportunities of quality informatics.

To calculate an eCQM, providers must have project management infrastructure to staff and run all levels necessary to calculate the metric. The provider must have an information system in place to collect, store, and analyze data, as well as write code to calculate the eCQM. The specifics are beyond the scope of this letter, but it suffices to say that there are technical components required to manage a healthcare quality information system (HQIS) that provides the data necessary for quality informatics. ${ }^{15}$ Quality informatics establishes and manages the necessary technical architecture to accurately handle data related to quality and concurrently connect with the project management and reporting necessary to make use of the system.

Reporting is a very visible component of quality informatics that encompasses quality metrics determined by institutions, payers, and governments. Quality informaticians are a critical part in determining the necessary metrics and understanding the capabilities of healthcare information systems. Metric reporting continues to gain importance with the increase in payments tied to quality metrics. ${ }^{4}$ Areas of emphasis in quality informatics also shift over time as quality metrics set by state and federal government agencies change their focus. For instance, CMS now emphasizes EHR interoperability, patient-provider data sharing, and patient access to records (reflected by the program name change from "Meaningful Use" to "Promoting Interoperability"12). Quality informatics is needed to allow the sharing and establishment of formal and effective approaches to each step described in the calculation of an eCQM.

\section{The How}

To have an effective quality informatics program, strong healthcare quality data and effective organizational structure are crucial to success. Niland et al described an effective program that uses a HQIS for effective healthcare quality information collection, management, analysis, and reporting. ${ }^{15}$ Each system component is divided into data, people, and procedural perspectives, which are key components of a quality informatics program. They especially note that the socio-technical and knowledge management aspects of a quality information system present challenges greater than the technical part of the system.

An organizational structure that supports quality improvement needs to be in place for effectively utilizing healthcare data for improvement. An informatician alone cannot complete the range of tasks required for an impactful quality informatics program. They must work with a team of analysts, data scientists, and information technology professionals. A culture of quality improvement must exist in the larger organization to achieve effective quality improvement results. In key informant interviews, Millery and Kukafka found a theme that healthcare information technology cannot be implemented effectively for quality without an underlying culture of quality improvement across the whole organization. ${ }^{16}$

The connection between healthcare quality metrics and healthcare service processes must be supported. Approaches like eCQMs alone will not improve quality; they must be incorporated into quality improvement processes to effect 
change. De Lusignan described how informatics forms a crucial component of the quality improvement process within England's National Health Service. ${ }^{17}$ By establishing the necessary HQIS, having the proper analytical tools, and building the organizational structure and culture, the foundation can be laid to build an effective quality informatics program that supports an organization's efforts to improve clinical effectiveness.

\section{The Opportunities}

Multiple opportunities present themselves for harnessing the capabilities and importance of quality informatics. The large amounts of healthcare data have given rise to the notion of "big data" in health care, characterized by the "five V's" (volume, velocity, variety, veracity, and value). ${ }^{18}$ The variety of data includes the many different data elements that come from many different patients served by many different providers, which in turn provide large quantities of data in realtime with varying quality that can be used to promote clinical excellence. Big data promises to improve the quality of healthcare delivery through the use of data analytics. ${ }^{19}$ As more data are collected, tools to support analysis and reporting continue to be developed, which will allow quality informaticians to gain more insight. For instance, commercial products enable healthcare systems to understand their quality of healthcare delivery. Using big data, predictive analytics also offers an opportunity to improve the quality of care if the right conditions are met for quality input data. ${ }^{20}$

Expanding the results of real-time analysis that are reported to clinicians as data are collected is a growing area of interest. For example, real-time quality data dashboards have been shown to impact the quality of care delivered in emergency departments and pediatric intensive care units. ${ }^{21,22}$ The continual development of EHRs, clinical big datasets, and sophisticated analytics coupled with reporting mechanisms holds promise for quality informatics to grow in its capabilities and opportunities to use information for the betterment of healthcare. As methodologies and techniques are developed that allow for the better use of healthcare data toward improving clinical effectiveness, quality informatics will serve as a home to incorporate these technologies into existing methodologies and distribute this knowledge to those who will benefit.

\section{The Challenges}

Quality informatics holds strong potential as a field to align with work done with different departments and fields to improve the quality of healthcare delivery. Designing an effective HQIS is a key component of a quality informatics program, but can be difficult. Challenges remain that can hinder the potential of achieving effective results, such as the quality, volume, and use of data from HQISs.

A great challenge quality informaticians are faced with is how to gather quality data. Sukumar et al have noted problems in the quality of large datasets in health care. ${ }^{23}$ Furthermore, data-quality issues are only addressed as they come at the moment. Defining, recognizing, and ensuring the quality of data are crucial. Without quality data, all further products of the system will potentially be compromised. A new approach is thus needed to systematically approach the quality of data. Organizations and informaticians must ensure the proper system is in place to supply the organization with qualified data. Data-quality assessment frameworks are being formalized for characterizing healthcare data quality for secondary uses. ${ }^{24-30}$ Notable challenges include development of metrics to assess data quality, ${ }^{24}$ definition of completeness users choose to use, ${ }^{25}$ and potential biases in datasets. ${ }^{26}$

Challenges facing systems and their attempts to use data for quality improvement include usability, interoperability, full integration, and data mining. ${ }^{31}$ In April 2018, CMS launched a request for information from providers to gather information that will inform quality measures based on the exchange of information between providers and patients. ${ }^{32}$ CMS also created its first Data Elements Library to place standards and data elements in one location to support the development of tools for interoperability. ${ }^{33}$

New methodologies are required to optimize data management, extract meaningful information, and conduct a proper workflow with the data because big data present new challenges due to the volume of data. ${ }^{19}$ As part of data governance, healthcare organizations need to ensure that the correct technology and staffing are in place for proper curation and maintenance of vast amounts of data to address their analytic needs. Each of big data's five V's poses a unique set of challenges that must be addressed to make effective use of available data. ${ }^{34}$ Unstructured data in the EHR (e.g., patient notes) present challenges and necessitate use of natural language processing (NLP) to transform freetext into structured data for subsequent analyses. For example, electronic quality (eQuality) assessment tools have been developed that incorporate NLP for automated quality measurement (e.g., for disability exams, ${ }^{35,36}$ postoperative complications, ${ }^{37}$ and asthma care ${ }^{38,39}$ ).

Data collection, analysis, and reporting cannot be separated from clinical practice and quality improvement programs on the ground. Informatics must be effectively tied to the larger organizational systems to truly achieve the goal of quality improvement. For quality metrics to be an effective part of quality improvement, they must be integrated into the whole system. As Burstin et al note, "To make significant improvements in U.S. health care, a closer connection between measurement and both evolving national data systems and evidence-based improvement strategies is needed." 40

Alongside the technical and organizational challenges of quality informatics, ethical questions require consideration. The collection of patient data raises questions of who owns those data, who has access, how the data are secured, and who regulates these data. Genomics faces similar ethical challenges surrounding the collection, use, and sharing of patient data. ${ }^{41}$ When patient data are incorporated into metrics, ethical questions emerge about patient selection for metrics, how the mission of healthcare providers changes when guided by metrics, and how the connection of monetary elements tied to metrics influences organizational decisions. Cohen et al discuss these ethical and legal challenges in the development and use of predictive analytics in health care. ${ }^{42}$ 
Each healthcare organization faces challenges to accomplish these tasks relative to their unique situations and organizational structures. How these organizations choose to develop an infrastructure to overcome these issues and maximize efficiency will depend on myriad factors, but effective leadership will be a key piece of any plan to effect change. For example, Tang et al showed that coding data contained in the EHR compared with manual chart review identified more diabetic patients correctly and led to significantly different quality measures without adding to the administrative burden. ${ }^{43}$ However, there are burdens associated with reporting quality measures, and research is needed to ensure that the amount of work that goes into reporting quality is then rewarded with a meaningful piece of information for that healthcare system to use. ${ }^{44}$ Informed and effective leadership is necessary to take these situations and translate examples from the literature into a meaningful reality for that organization. Leadership needs to develop a plan that guides data from collection to reporting in a smooth pipeline and connect that with the systems for improvement. Without key leadership, these areas have the potential to function as independent silos, wasting resources and potentially reducing impact.

\section{Conclusion}

Healthcare systems are accountable for monitoring, reporting, and improving the quality of clinical care. As the need for reporting and healthcare data grows, quality informatics is needed to coordinate and manage quality improvement projects, HQISs, technical components of information systems, and the reporting of data for promoting clinical excellence. Although institutions face challenges to develop effective quality informatics programs, quality informatics presents growing opportunities. Quality informatics enables working groups and guidelines to be developed for supporting coordination and sharing of work to improve healthcare delivery.

\section{Clinical Relevance Statement}

Quality informatics aims to utilize the information contained in healthcare information systems to help healthcare practitioners achieve improvements in clinical effectiveness. As healthcare information systems continue to grow, reporting requirements continue, and payment models move toward an emphasis on quality metrics, quality informatics is needed to coordinate and support the work done to achieve these results.

\section{Multiple Choice Questions}

1. What are the components of the work of quality informatics?

a. Quality improvement programs, electronic health record management, and data analysis

b. Healthcare quality metric calculations and quality improvement program management c. Project management, technical management, and reporting

d. Technical infrastructure management, healthcare quality metric calculations, and reporting

Correct Answer: The correct answer is option c, project management, technical management, and reporting. Quality informatics encompasses the gathering of information, processing of information, and reporting of information toward improving clinical effectiveness. Project management is necessary to ensure that the proper organizational infrastructure exists to gather, analyze, and then utilize the information from a healthcare quality information system. Technical management ensures that the healthcare quality information system can adequately gather and analyze the data needed, and reporting of quality metrics and outcomes is a growing requirement to payers and government agencies and these metrics can also be used for internal quality improvement programs.

2. What are some of the greatest challenges facing quality informatics?

a. Construction of an effective healthcare quality information system, data analysis, and quantity of metrics to be reported

b. Gathering quality data, analyzing large volumes of data, and connecting data to quality improvement programs

c. Technical difficulty of data analysis, employee investment in quality improvement programs, and effective project management

d. Effective input of data into a healthcare quality information system and ease of data extraction from the healthcare quality information system

Correct Answer: The correct answer is option b, gathering quality data, analyzing large volumes of data, and connecting data to quality improvement programs. To have data to analyze, data must first be put into and gathered from an effective and efficient healthcare quality information system. Developing this system requires multiple technical and human resources that must be intentionally designed and trained. The quantity of healthcare data now stored in electronic health records presents technical challenges to analyze such large quantities of data. Once collected and analyzed, for quality informatics to actually improve clinical effectiveness, the information gathered cannot be separated from the operational processes in the healthcare setting. The data need to be connected to quality improvement programs to allow the data to help inform the improvement programs and for the programs to help improve the quality metrics.

\section{Protection of Human and Animal Subjects}

Human and/or animal subjects were not included in the project.

\section{Funding}

This work was supported in part by the Brown Center for Biomedical Informatics and Institutional Development 
Award Number U54GM115677 from the National Institute of General Medical Sciences of the National Institutes of Health, which funds Advance Clinical and Translational Research (Advance-CTR). The content is solely the responsibility of the authors and does not necessarily represent the official views of the National Institutes of Health.

\section{Conflict of Interest}

None declared.

\section{References}

1 Kruse CS, Kothman K, Anerobi K, Abanaka L. Adoption factors of the electronic health record: a systematic review. JMIR Med Inform 2016;4(02):e19

2 Weiner JP, Fowles JB, Chan KS. New paradigms for measuring clinical performance using electronic health records. Int J Qual Health Care 2012;24(03):200-205

3 Simpao AF, Ahumada LM, Gálvez JA, Rehman MA. A review of analytics and clinical informatics in health care. J Med Syst 2014; 38(04):45

4 Keswani A, Uhler LM, Bozic KJ. What quality metrics is my hospital being evaluated on and what are the consequences? J Arthroplasty 2016;31(06):1139-1143

5 OHSU Clinfowiki. Quality informatics. Available at: http://clinfowiki.org/wiki/index.php/Quality_informatics. Accessed June, 2016

6 Kulikowski CA, Shortliffe EH, Currie LM, et al. AMIA Board white paper: definition of biomedical informatics and specification of core competencies for graduate education in the discipline. J Am Med Inform Assoc 2012;19(06):931-938

7 AMIA. Clinical Informatics. Available at: https://www.amia.org/ applications-informatics/clinical-informatics. Accessed June, 2016

8 Safran C, Bloomrosen M, Hammond WE, et al; Expert Panel. Toward a national framework for the secondary use of health data: an American Medical Informatics Association White Paper. J Am Med Inform Assoc 2007;14(01):1-9

9 Elkin PL, Trusko BE, Koppel R, et al. Secondary use of clinical data. Stud Health Technol Inform 2010;155:14-29

10 Hripcsak G, Bloomrosen M, FlatelyBrennan P, et al. Health data use, stewardship, and governance: ongoing gaps and challenges: a report from AMIA's 2012 Health Policy Meeting. J Am Med Inform Assoc 2014;21(02):204-211

11 Peterson KJ, Pathak J. Scalable and high-throughput execution of clinical quality measures from electronic health records using MapReduce and the JBoss ${ }^{\circledR}$ drools engine. AMIA Annu Symp Proc 2014;2014:1864-1873

12 CMS.gov. CMS proposes changes to empower patients and reduce administrative burden. Available at: https://www.cms.gov/news$\mathrm{room} /$ press-releases/cms-proposes-changes-empower-patientsand-reduce-administrative-burden. Accessed October 27, 2018

13 Dixon-Woods M, Redwood S, Leslie M, Minion J, Martin GP, Coleman JJ. Improving quality and safety of care using "technovigilance": an ethnographic case study of secondary use of data from an electronic prescribing and decision support system. Milbank Q 2013;91(03):424-454

$14 \mathrm{Mo} \mathrm{H}$, Pacheco JA, Rasmussen LV, et al. A prototype for executable and portable electronic clinical quality measures using the KNIME analytics platform. AMIA Jt Summits Transl Sci Proc 2015; 2015:127-131

15 Niland JC, Rouse L, Stahl DC. An informatics blueprint for healthcare quality information systems. J Am Med Inform Assoc 2006;13 (04):402-417

16 Millery M, Kukafka R. Health information technology and quality of health care: strategies for reducing disparities in underresourced settings. Med Care Res Rev 2010;67(5, Suppl):268S-298S
17 de Lusignan S. Informatics as tool for quality improvement: rapid implementation of guidance for the management of chronic kidney disease in England as an exemplar. Healthc Inform Res 2013;19(01):9-15

18 Baro E, Degoul S, Beuscart R, Chazard E. Toward a literature-driven definition of big data in healthcare. BioMed Res Int 2015; 2015:639021

19 Luo J, Wu M, Gopukumar D, Zhao Y. Big data application in biomedical research and health care: a literature review. Biomed Inform Insights 2016;8:1-10

20 Janke AT, Overbeek DL, Kocher KE, Levy PD. Exploring the potential of predictive analytics and big data in emergency care. Ann Emerg Med 2016;67(02):227-236

21 Stone-Griffith S, Englebright JD, Cheung D, Korwek KM, Perlin JB. Data-driven process and operational improvement in the emergency department: the ED Dashboard and Reporting Application. J Healthc Manag 2012;57(03):167-180

22 Shaw SJ, Jacobs B, Stockwell DC, Futterman C, Spaeder MC. Effect of a real time pediatric ICU safety bundle dashboard on quality improvement measures. Jt Comm J Qual Patient Saf 2015;41(09): $414-420$

23 Sukumar SR, Natarajan R, Ferrell RK. Quality of big data in health care. Int J Health Care Qual Assur 2015;28(06):621-634

24 Weiskopf NG, Weng C. Methods and dimensions of electronic health record data quality assessment: enabling reuse for clinical research. J Am Med Inform Assoc 2013;20(01): 144-151

25 Weiskopf NG, Hripcsak G, Swaminathan S, Weng C. Defining and measuring completeness of electronic health records for secondary use. J Biomed Inform 2013;46(05):830-836

26 Rusanov A, Weiskopf NG, Wang S, Weng C. Hidden in plain sight: bias towards sick patients when sampling patients with sufficient electronic health record data for research. BMC Med Inform Decis Mak 2014; $14: 51$

27 Weiskopf NG, Bakken S, Hripcsak G, Weng C. A data quality assessment guideline for electronic health record data reuse. EGEMS (Wash DC) 2017;5(01):14

28 Callahan TJ, Bauck AE, Bertoch D, et al. A comparison of data quality assessment checks in six data sharing networks. EGEMS (Wash DC) 2017;5(01):8

29 Lee K, Weiskopf N, Pathak J. A framework for data quality assessment in clinical research datasets. AMIA Annu Symp Proc 2018;2017:1080-1089

30 Kahn MG, Callahan TJ, Barnard J, et al. A harmonized data quality assessment terminology and framework for the secondary use of electronic health record data. EGEMS (Wash DC) 2016;4(01): 1244

31 Weigel FK, Switaj TL, Hamilton J. Leveraging health information technology to improve quality in federal healthcare. US Army Med Dep J 2015;(Oct-Dec):68-74

32 CMS.gov. Fiscal year (FY) 2019 Medicare hospital inpatient prospective payment system (IPPS) and long term acute care hospital (LTCH) prospective payment system proposed rule, and request for information. Available at: https://www.cms.gov/newsroom/fact-sheets/fiscal-year-fy-2019-medicare-hospital-inpatient-prospective-payment -system-ipps-and-long-term-acute. Accessed November 2, 2018

33 CMS.gov. CMS launches data element library supporting interoperability. Available at: https://www.cms.gov/newsroom/pressreleases/cms-launches-data-element-library-supporting-interoperability. Accessed November 2, 2018

34 Anuradha J. A brief introduction on big data 5Vs characteristics and Hadoop technology. Procedia Comput Sci 2015;48:319-324

35 Brown SH, Speroff T, Fielstein EM, et al. eQuality: electronic quality assessment from narrative clinical reports. Mayo Clin Proc 2006;81(11):1472-1481

36 Brown SH, Elkin PL, Rosenbloom ST, Fielstein E, Speroff T. eQuality for all: extending automated quality measurement of free text clinical narratives. AMIA Annu Symp Proc 2008;2008:71-75 
37 Murff HJ, FitzHenry F, Matheny ME, et al. Automated identification of postoperative complications within an electronic medical record using natural language processing. JAMA 2011;306(08):848-855

38 Hazlehurst B, McBurnie M, Mularski R, Puro J, Chauvie S. Automating quality measurement: a system for scalable, comprehensive, and routine care quality assessment. AMIA Annu Symp Proc 2009;2009:229-233

39 Hazelhurst B, McBurnie MA, Mularski RA, Puro JE, Chauvie SL. Automating care quality measurement with health information technology. Am J Manag Care 2012;18(06):313-319

40 Burstin H, Leatherman S, Goldmann D. The evolution of healthcare quality measurement in the United States. J Intern Med 2016; 279(02):154-159
41 Knoppers BM, Thorogood AM. Ethics and big data in health. Curr Opin Syst Biol 2017;4:53-57

42 Cohen IG, Amarasingham R, Shah A, Xie B, Lo B. The legal and ethical concerns that arise from using complex predictive analytics in health care. Health Aff (Millwood) 2014;33(07):1139-1147

43 Tang PC, Ralston M, Arrigotti MF, Qureshi L, Graham J. Comparison of methodologies for calculating quality measures based on administrative data versus clinical data from an electronic health record system: implications for performance measures. J Am Med Inform Assoc 2007;14(01):10-15

44 Rudin RS, Tang PC, Bates DW. Health information technology policy. In: Shortliffe E, Cimino J, eds. Biomedical Informatics. London: Springer; 2014 